\title{
Rib spacing effect on heat transfer and pressure loss in a rectangular channel with semicircular ribs
}

\author{
Prakash S. Patil* and K. K. Dhande** \\ * Research scholar, Department of Mechanical Engineering, Rajarshi shahu college of Engineering, Pune, India. \\ ** Prof. \& Head, Department of Mechanical Engineering, D.Y.Patil Institute of Technology, Pune, India. \\ *Corresponding Author: prakashrane35@gmail.com
}

Submitted : 19/06/2020

Revised :24/10/2020

Accepted : 28/11/2020

\begin{abstract}
Ribs of various shapes are used for heat transfer enhancement, but its performance significantly depends on geometrical features and flow conditions. This study experimentally finds out the influence of rib spacing, and semicircular shape ribs with three-rib spacing ratio $(\mathrm{P} / \mathrm{e})=8,10$, and 12 are studied and located on lower wall of the rectangular channel. Reynolds numbers varied from 10000 to 29,000 and the blockage ratio of the channel (e/Dh) was 0.151 . The results show that semicircular rib performed better than plain plate, but more friction is found. Semicircular rib with rib spacing of $50 \mathrm{~mm}(\mathrm{P} / \mathrm{e}=10)$ shows highest thermal performance, enhanced by an average of $39 \%$ heat transfer than rib spacing of 40 and $60 \mathrm{~mm}$ (P/e=8 and 12). Friction losses were observed to be the highest in rib spacing ratio of 8 and found an average of $10 \%$ more friction compared to rib spacing ratio of 10 and 12 . Semicircular rib with spacing ratio 8 shows the least thermal performances compared to other configurations.
\end{abstract}

Keywords: Semicircular rib; Heat transfer enhancement; Rib spacing; Rectangular channel.

\begin{tabular}{|c|l|c|}
\hline \multicolumn{2}{|c|}{ Nomenclature } \\
\hline Symbol & \multicolumn{1}{|c|}{ Description } & Units \\
\hline$A$ & rectangular duct cross section area & $\mathrm{m}^{2}$ \\
\hline$h$ & heat transfer coefficient & $\mathrm{W} / \mathrm{m}^{2} \mathrm{~K}$ \\
\hline$D_{h}$ & Rect. duct hydraulic diameter & $\mathrm{m}$ \\
\hline$W$ & Width of rect. duct & $\mathrm{m}$ \\
\hline$H$ & height of rect. duct & $\mathrm{m}$ \\
\hline$L$ & Test section length & $\mathrm{m}$ \\
\hline$p$ & Rib pitch & $\mathrm{m}$ \\
\hline$e$ & Rib height & $\mathrm{m}$ \\
\hline
\end{tabular}




\begin{tabular}{|c|l|c|}
\hline$m$ & Air mass flow rate & $\mathrm{kg} / \mathrm{s}$ \\
\hline$A R$ & Channel aspect ratio $(\mathrm{W} / \mathrm{H})$ & \\
\hline$p / e$ & Ratio of rib pitch to rib height & $\mathrm{W}$ \\
\hline$Q_{n e t}$ & Net Heat gain & $0^{\mathrm{C}}$ or $\mathrm{K}$ \\
\hline$T_{i}$ & Inlet temperature of air & $0^{\mathrm{C}}$ or $\mathrm{K}$ \\
\hline$T_{o}$ & outlet temperature of air & \\
\hline \multicolumn{1}{|c|}{ Dimensionless number } \\
\hline$R e$ & Reynolds number & \\
\hline$N u$ & Nusselt number & \\
\hline$N u_{o}$ & smooth duct Nusselt number & \\
\hline$f$ & Friction factor & \\
\hline$f o$ & Friction factor for smooth duct & \\
\hline
\end{tabular}

\section{INTRODUCTION}

The main goal of every industry is to achieve the maximum efficiency by using latest techniques, the same as the case in gas turbine engines to get more efficiency and output where it is required to operate at maximum inlet temperature. This high inlet temperature was not sustained by blade material; to save life of turbine blade, cooling is necessary. The internal cooling method consists of pin fin cooling, rib turbulators, dimple, protrusions, etc. Generally, ribs ae preferred for cooling, and the performance of rib turbulators depends on various factors like rib angle, blockage ratio, pitch ratio, rib shape, an inclination of rib, and the stationary or rotating condition. In the past decades, many researchers reported on various kinds of rib turbulators for heat transfer enhancement. Luca Baggetta studied 45degree angled rib configuration with intersecting ribs and concluded that, due to the presence of intersecting ribs, friction increased compared with heat transfer coefficient, as interesting ribs increased friction and were found to be more (Luca Baggetta et al. 2018). Kaewchoothong reported inclined ribs using the thermal liquid crystal sheet and concluded that Nusselt number for $60^{\circ}, 45^{\circ}$ angled ribs and $60^{\circ} \mathrm{V}$-shaped ribs has increased by 20 to $30 \%$ than $90^{\circ}$ angled ribs (Kaewchoothong et al. 2017). Truncation ribs were studied by Liu at Reynolds number 80,000, and it is concluded that truncated ribs reduced the friction without decreasing the heat transfer rate (Jian Liu et al. 2018). Some investigators focused on numerical techniques. Moon numerically studied sixteen rib shapes at Reynolds numbers of 5000-50,000. The Reynolds stress model was used, and it is concluded that the boot-shaped rib shows highest performance (Mi-Ae Moon et al. 2014). Zheng, using simulation, finds out the influence of rib arrangements on the thermal performance with V-type and parallel ribs, and the result showed that thermal performance of V-type ribs was highest compared to P-type ribs (Zheng et al. 2016). Shukla presented $90^{\circ}$ continuous, V -broken attached thick and thin ribs. It is shown that $90^{\circ}$ attached ribs show highest enhancement at rib spacing ratio (P/e) 10, and also the performance of thin ribs was improved by thick ribs (Anuj Shukla and Anupam Dewan 2016). Xie numerically reported various offset mid-truncated ribs at Reynolds number ranging from 10,000 to 50,000. Truncation ratio kept $12 \%$ in the middle and concluded that, due to truncated ribs, the heat transfer improved with reduced pressure loss penalty (Gongnan Xie et al. 2014). Wandong Bai numerically studied the entrance effect of rib induced vortices in pin fin array. The SST k- $\omega$ turbulence model was used, and it showed that the $90 \mathrm{deg}$. rib had the best overall performance (Wandong Bai et al. 2019).

Few researchers focused on rib spacing. Ansari reported the rib spacing effect at entry length of solar air heater. It is found that the nonuniform ribs with an increased pitch shows better thermal performances (Mohammad Ansari 
et al. 2020). Tanda experimentally studied the effect of rib spacing using 45 angled rib and reported different rib spacing ratio $(\mathrm{P} / \mathrm{e}=6.66,10,13.33,20)$ at Reynolds number from 9000 to 35000 . It is concluded that rib pitch ratio at 13.33 performed best for one ribbed wall channel and 6.66-10 for double ribbed wall (Govanni Tanda 2011, 2009). Yang experimentally studied three rib spacing ratios of 5, 10, and15 at Reynolds number from 1400 to 9000 . It is found that the rib spacing ratio 10 performed well compared to 5 and 15 spacing ratios, and also symmetric ribs performed better compared to staggered ribs (Yang et al. 2017). Guoqiang studied the effect of rib spacing at the pitch ratios 3.8 to 14.4 on the pressure side and 10 on the suction side in a rotating channel. The results show that, in positive direction at rib spacing ratio 3.8 , it achieved more enhancements, and in negative direction at rib spacing ratio 10, it indicates the good performance (Guoqiang Xu et al. 2015). Chaube experimentally studied the gap effect in inclined ribs and showed that the highest performance was achieved when the rib has a relative gap width of 1.0 at a relative gap position of $1 / 3$ (Chaube et al.2014).

Recently, some authors focused on newly developed ribs. Structured miniature and conventional ribs were presented by Yanlin, and they show that the performance of miniature structured ribs was higher than that of the conventional ribs; also, W shape rib performed better compared to other tested ribs (Yanlin Li et al. 2019). Sharma focused on pentagonal ribs and measured surface temperature distribution using liquid crystal thermography. It is concluded that the pentagonal ribs performed well than square ribs, and also it is reported that solid and converging slit ribs using liquid crystal thermography show that converging-slit ribs considerably enhance the heat transfer rate in the downstream vicinity (Naveen Sharma et al. 2017 \& 2018). Alfarawi focused on hybrid ribs and showed that the hybrid ribs performed well compared with the rectangular and semicircular ribs (S. Alfarawi et al. 2017). From the literature review, it was observed that less research work was found on rib spacing using semicircular shape ribs. Previous researchers are mostly focused on parallel, angled, and V-shaped ribs with spacing, and only few authors focused on circular type ribs, but they can not focus more on rib spacing, so there is still scope to work on it. The objective of the present study is to find new cooling method for gas turbine blade, which is essentially the optimum rib spacing of semicircular rib. In the proposed research, we experimentally find out the heat transfer characteristics and friction factor of semicircular shape ribs with three rib spacing ratio $(\mathrm{P} / \mathrm{e}) \mathrm{8}, 10$, and 12.

\section{EXPERIMENTATION}

\section{Experimental Facility}

To collect experimental data, experimental setup is designed, fabricated, and validated. Fig.1 shows schematic of experimental setup; it consists of centrifugal blower, developing section, an orifice plate with $U$ tube manometer, inlet section, rectangular channel with test section, exit section, k-type thermocouples, and data acquisition system.

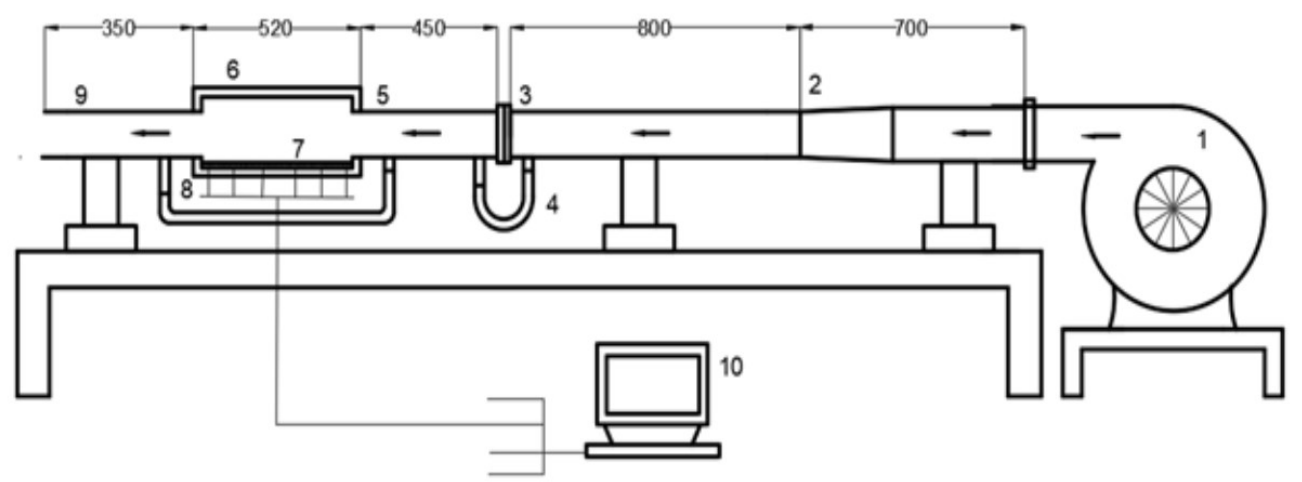

Figure 1. Schematic of experimental test facility. 
1-blower,

2- developing section

$3 \& 4$ - orifice plate \& U tube manometer
5- Inlet section

6- Rectangular channel

7- Test section
8- Thermo-couples

9 - Exit section

10- data acquisition system

In experimental setup, the blower was followed by developing section; the total length of this section is $1500 \mathrm{~mm}$, and the length kept more to ensure fully developed flow and regularize the flow. Developing section is followed by inlet section of length more than 10 times the hydraulic dia. of channel $\left(\mathrm{D}_{\mathrm{h}}\right)$, where again the flow becomes uniform and regularized. Mass flow rate of air was controlled by dimmer-stat and measured using orifice plate assembly. Rectangular channel was placed in between the inlet and the exit section and made from Bakelite material to reduce heat conduction loss. Aspect ratio of rectangular channel was 4:1, hydraulic diameter $\left(D_{h}\right)$ was 30 $\mathrm{mm}$, and length of channel was $540 \mathrm{~mm}$. In rectangular channel test section, base plate and heater were inserted, with the exit section of length more than 6 times the hydraulic dia. of channel $\left(\mathrm{D}_{\mathrm{h}}\right)$ being attached at the end to reduce the end effects of flow. Fig. 2 shows actual photograph of orifice plate and blower.
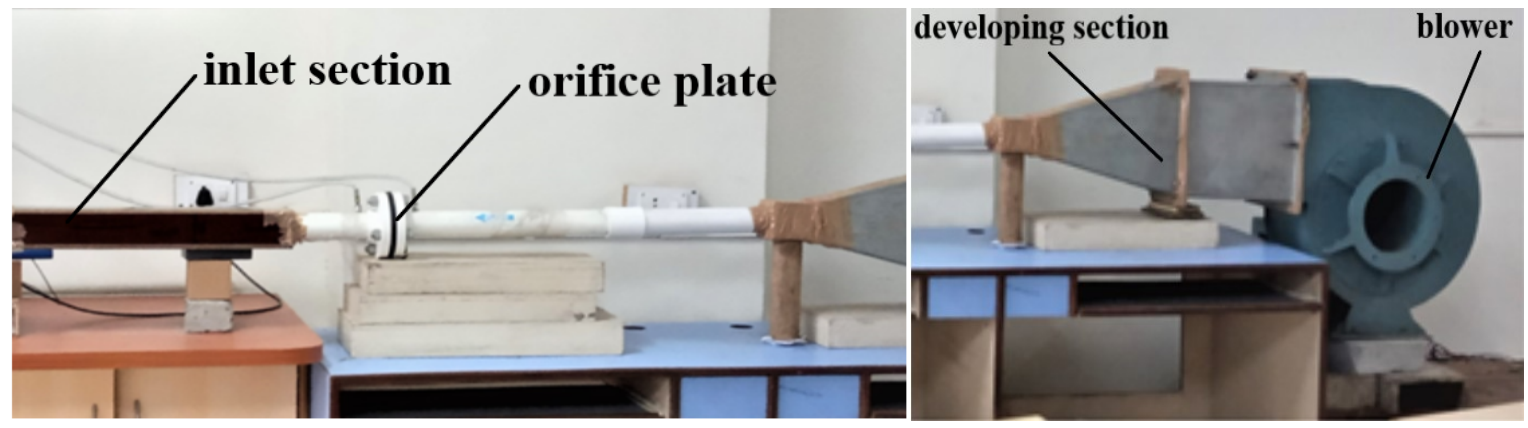

Figure 2. Photographs of experimental setup.

Pressure drop along the rectangular channel was recorded using a micro manometer connected along inlet and outlet of the test plate. Air inlet and outlet temperature are measured using two thermocouples located at inlet and exit side of duct and connected to data logger. Twelve thermocouples are provided at the surface of the test plate to measure the temperature of test section, and copper constant K-type thermocouples are used for temperature measurement. The uniform heat flux was supplied by the flat plate heater of size $300 \times 50 \mathrm{~mm}$ and inserted between the test plate and base plate. Heat input was varied with the help of dimmer-stat, and all instruments used for experimentation are calibrated from NABL accredited laboratory. At steady-state conditions, all data were recorded initially; it takes 2 to 3 hours.

\section{Test Section and Rib Configurations}

The material used for test section was aluminum, and thickness and length of the test plate were $10 \mathrm{~mm}$ and 540 $\mathrm{mm}$. The semicircular rib was pasted on the test plate using thermal glue. The test plate along with a heater and the supporting plate was tightly fastened with nut and bolts and placed in a rectangular channel. In order to reduce the heat losses, the bottom surface of heater and side wall are insulated with the insulating material.

Rib configurations. The ribs are pasted to a test section using bonding material. Semicircular shape ribs are manufactured from $75 \mathrm{~mm}$ diameter aluminum pipe. Aluminum pipe is first made hollow using a boring tool and then prepared outside diameter of the pipe in the correct size on lathe machine; after that, on a special purpose 
machine, the round ribs are cut according to the required size. The height and width of the rib are $5 \mathrm{~mm}$. Height of rib to channel hydraulic dia. ratio $\left(\mathrm{e} / \mathrm{D}_{\mathrm{h}}\right)=0.156$, and ratio of rib pitch to rib height $(\mathrm{P} / \mathrm{e})$ was 8,10 , and 12 . Fig. 3 shows actual photograph of rectangular channel with test plate.

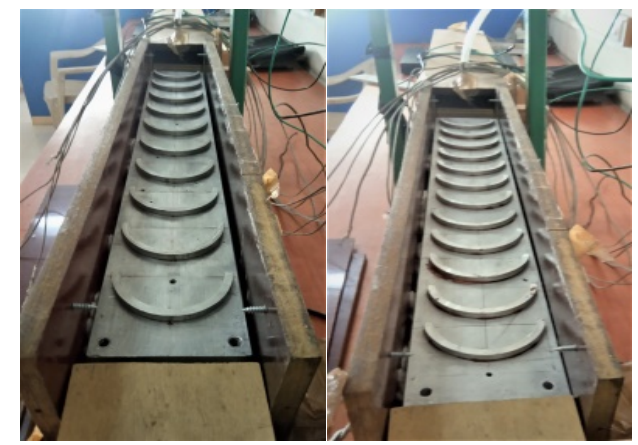

Figure 3. a) Rectangular duct with test section assembly (10 ribs and 12 ribs).

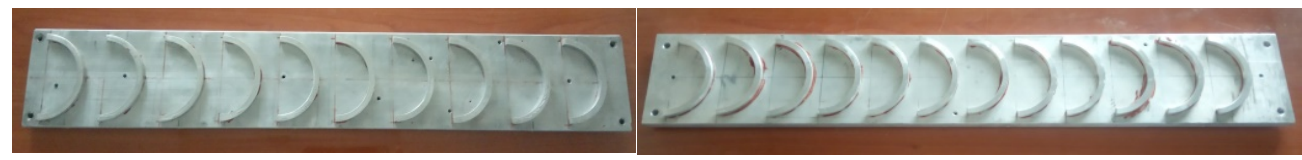

Figure 3. b) Semicircular rib $(\mathrm{P} / \mathrm{e}=10 \& 8)$.

Rib arrangement. Three ribbed channels are prepared for testing with different rib spacing. In the first set of semicircular ribs having rib spacing ratio $(\mathrm{P} / \mathrm{e})=8$ studied, here, the distance between two ribs is $40 \mathrm{~mm}$. In the second set, the same ribs are studied, but the pitch of rib to height of rib ratio $(\mathrm{P} / \mathrm{e})=10$, which means that the distance between two ribs is $50 \mathrm{~mm}$, and in the third set, rib spacing ratio $(\mathrm{P} / \mathrm{e})$ was kept at 12 , which means that the distance between two ribs is $60 \mathrm{~mm}$. Ribs are tested at Reynolds number from 10000 to 29000 and placed on the lower surface of channel, and the aspect ratio of channel was 4:1.

\section{DATA REDUCTION AND ERROR ANALYSIS}

The recorded data of temperatures and pressures at different places in the channel are used to find out various parameters such as friction factor and its ratio; Nusselt number and its ratio, and thermal performance. The heat transfer coefficient (ha) was calculated by equation (1):

$$
\begin{aligned}
\mathrm{Q}_{\text {net }} & =\mathrm{ha} \mathrm{A}(\mathrm{Ts}-\mathrm{Tb}) \\
\mathrm{h} & =\frac{\mathrm{Q}_{\text {net }}}{\mathrm{A}\left(\mathrm{T}_{\mathrm{s}}-\mathrm{T}_{\mathrm{b}}\right)}-------- \\
T S & =\frac{\mathrm{T}_{1}+\mathrm{T}_{2}+----+\mathrm{T}_{12}}{12}, T_{b}=\frac{\mathrm{T}_{\text {air in }}+\mathrm{T}_{\text {air out }}}{2}
\end{aligned}
$$

where $T s$ is average surface temperature of test plate, and $\mathrm{T}_{\mathrm{b}}$ is bulk temperature of air. Net heat input was calculated considering account heat losses from the test plate (conduction, convection), and the maximum heat loss from the test section was calculated to be less than $12 \%$. Nusselt number was calculated using equation (2), where $h$ 
is the average heat transfer coefficient, $\mathrm{Dh}$ is the hydraulic diameter of channel, A is the cross section area of channel, and $\mathrm{p}$ is the perimeter of channel:

$$
\begin{aligned}
& \mathrm{Nu}=\frac{\mathrm{h} \mathrm{D}_{\mathrm{h}}}{\mathrm{k}} \\
& D_{h}=\frac{4 \mathrm{~A}}{\mathrm{p}}
\end{aligned}
$$

The Dittus-Boelter correlation was applied to validate the Nusselt number of plain plate. The Nusselt number ratio is given as

$$
\mathrm{Nu} / \mathrm{Nu}_{\mathrm{o}}=(\mathrm{hDh} / \mathrm{k}) /\left(0.023 \operatorname{Re}^{0.8} \operatorname{Pr}^{0.4}\right)
$$

Frictional Losses. The friction factor was calculated by measuring pressure drop along the test channel and calculate using equation (4):

$$
\begin{aligned}
& \Delta \mathrm{P}=\mathrm{fL}_{\rho} \mathrm{V}^{2} \text { avg./2 D } \\
& \mathrm{f}=\left(\mathrm{P}_{\mathrm{i}}-\mathrm{Pe}\right) /\left[\left(4\left(\mathrm{~L} / \mathrm{D}_{\mathrm{h}}\right)\left(1 / 2 \rho \mathrm{V}^{2}\right)\right]\right.
\end{aligned}
$$

The frictional factor ratio was calculated by considering friction factor of smooth plate. The Blasius correlation validates friction factor values of smooth plate.

$$
\mathrm{f} / \mathrm{f}_{0}=\mathrm{f} /\left(0.079 \mathrm{Re}^{-1 / 4}\right)
$$

Thermal Performance. Thermal hydraulic performance was calculated by taking into account the Nusselt number ratio $(\mathrm{Nu} / \mathrm{Nuo})$ and the frictional factor ratio (f/fo). Equation (6) was used to evaluate the performance ( $\mathrm{\eta})$ of rib configuration.

$$
\eta=\left(\mathrm{Nu} / \mathrm{Nu}_{0}\right) /\left(f / f_{0}\right)^{1 / 3}
$$

Error analysis: the Kline and McClintock (1953) method was used for uncertainty analysis; the calculated uncertainty in the temperature measurement was found to be \pm 10 for all cases. In the Nusselt number, the average uncertainty was almost $8-11 \%$ of the experimental values. Initially, more heat loss was observed at low Reynold's number because more uncertainty was observed, but as flow velocity increased, uncertainty reduced. In the friction factor, the overall uncertainty was almost $7-10 \%$ of the experimental values.

\section{Heat Transfer in Smooth Duct}

The experimental results of smooth duct are validated using correlation results. Fig. (4) presents experimental result of Nusselt number with result achieved from Dittus-Boelter correlation (7) for plain duct. A better relation was seen when linking the experimental results of Nusselt number with the correlations results, and a maximum of $13 \%$ deviation was observed in Nusselt number between the experimental and correlation values. Experimental friction factor value correlates with values obtained from Modified Blasius equation (8) for plain duct. Meantime, the friction factor of smooth duct correlates closely with Blasius's correlation as presented in Fig. (5), observed highest $10 \%$ difference in the theoretical and experimental value of friction factor. 


$$
\begin{aligned}
& \mathrm{Nu}_{\mathbf{o}}=0.023 \mathrm{Re}^{0.8} \operatorname{Pr}^{0.4} \\
& \mathrm{f}_{\mathbf{0}}=0.079 \mathrm{Re}^{-\mathbf{1} / 4}
\end{aligned}
$$

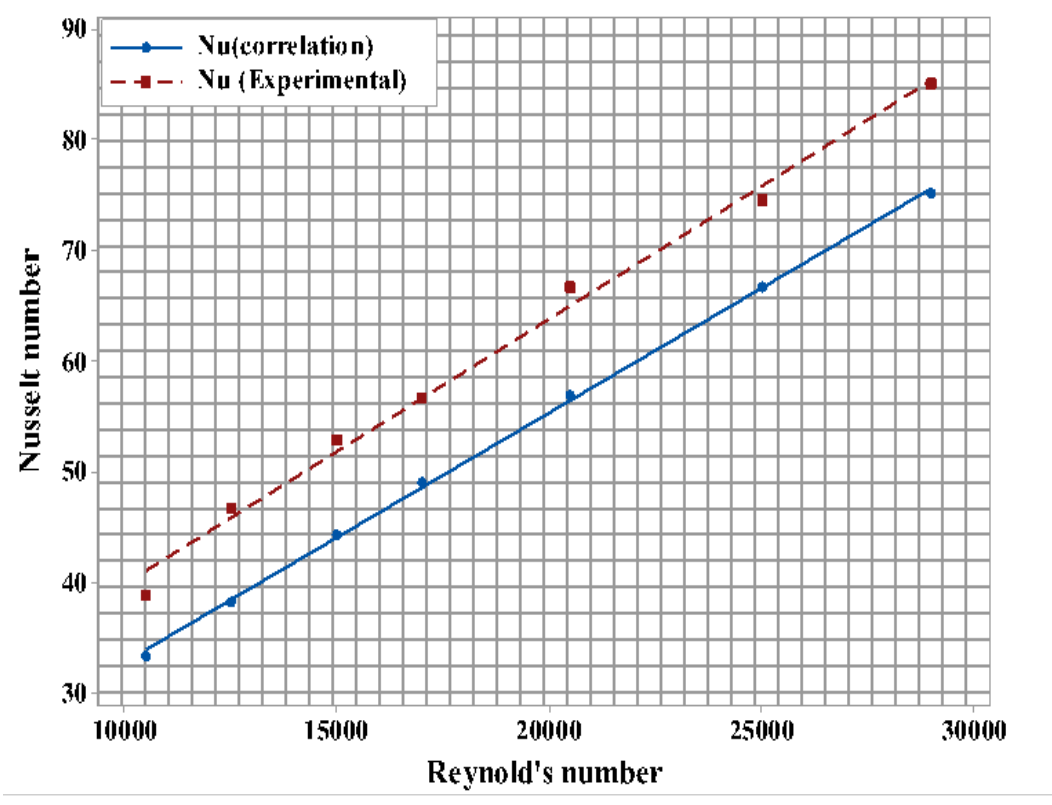

Figure 4. Comparison of smooth duct results of Nusselt number with correlation.

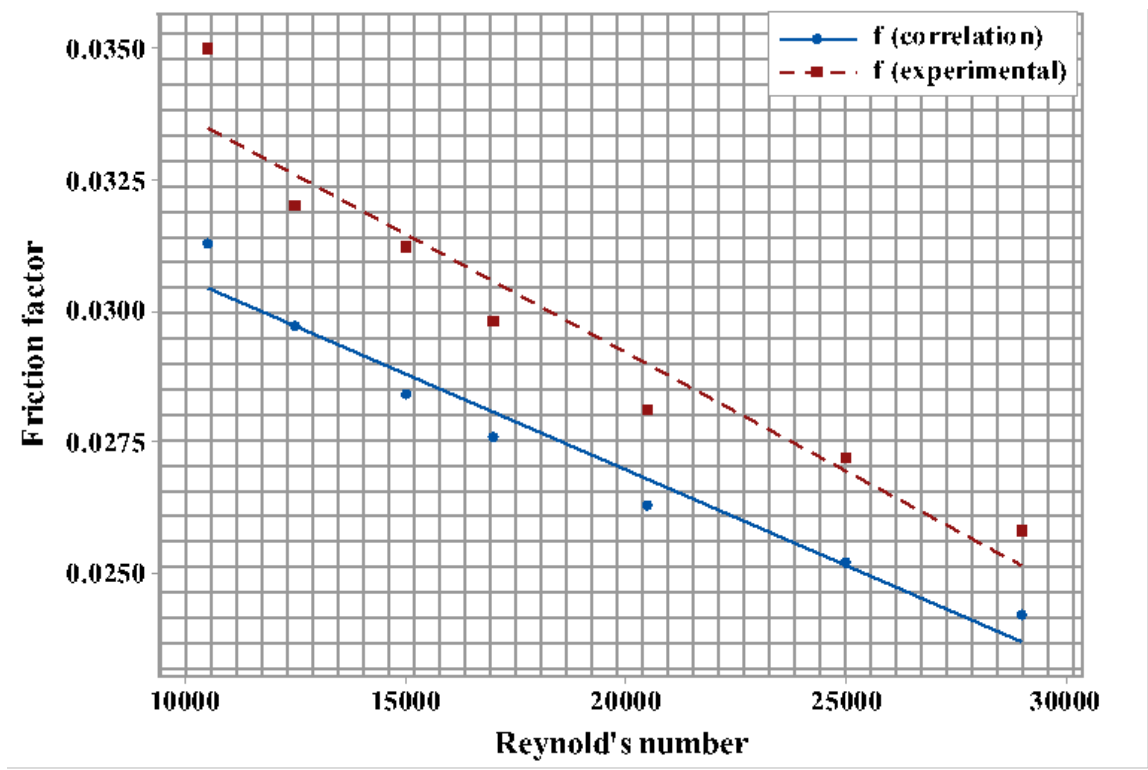

Figure 5. Comparison of smooth duct results with correlation. 


\section{RESULTS AND DISCUSSION}

Here, the Nusselt number and friction factor of all tested configurations are presented and discussed at a range of Reynolds number 10000 to 29000 . The results of different configurations have been studied and compared with each other to find the optimum rib spacing for semicircular rib.

\section{Heat Transfer in Ribbed Duct}

From the experimental data, the Nusselt number of semicircular rib was calculated at various rib spacings and is shown in Fig. (6). The Nusselt number for all channels shows increasing trend means, and as the Reynolds number increased, the Nusselt number also increased. From the graph, it can also be seen that the semicircular rib performs well compared to the plain plate, enhanced up to $130 \%$ heat transfer compared to plain plate. Application of ribs on the surface increased the heat transfer area and also increased separation of rib turbulators on flow boundary layer, and due to this reason, the rib increased heat transfer, but due to obstruction pressure drop created. Semicircular shape ribs have more frontal area due to curved shape and also create reduced heat transfer area behind the ribs because this heat transfer increased. In case of semicircular rib, high turbulent kinetic energy appears at the middle region between two adjacent ribs, and in front of the ribs, it was also observed that the fluid recirculation region with low turbulent kinetic energy and velocity appears behind the ribs.

From the figure, it is also indicated that, for semicircular shape rib, the Nusselt number was highest at rib pitch to height ratio $(\mathrm{P} / \mathrm{e})=10$, after $\mathrm{P} / \mathrm{e}=8$ and 12 follows at all Reynold's number, which means that the semicircular rib with spacing ratio 10 performs better compared to other tested configurations. In the case of $40 \mathrm{~mm}$ rib pitch $(\mathrm{P} / \mathrm{e}=8)$, spacing between the two ribs observed was less because the flow reattachment to the rib wall will be slowly reduced, as the falling of the rib pitch and the phenomena of flow reattachment were not developed; thus, it reduced the heat transfer rate at the rib pitch ratio $(\mathrm{P} / \mathrm{e}=8)$. In the case of $60 \mathrm{~mm}$ rib pitch $(\mathrm{P} / \mathrm{e}=12)$, spacing between two ribs was observed more because the boundary layer grows later reattachment points and also the reattachment point at the wall was reached, and a boundary layer starts to develop early, and the subsequent rib comes across; thus, it reduced the heat transfer rate at the rib pitch ratio $(\mathrm{P} / \mathrm{e}=12)$, but more than $40 \mathrm{~mm}$ rib spacing. However, with the increase of the rib spacing, the effects of separation vortex and reattachment on convective heat transfer are weaker and weaker.

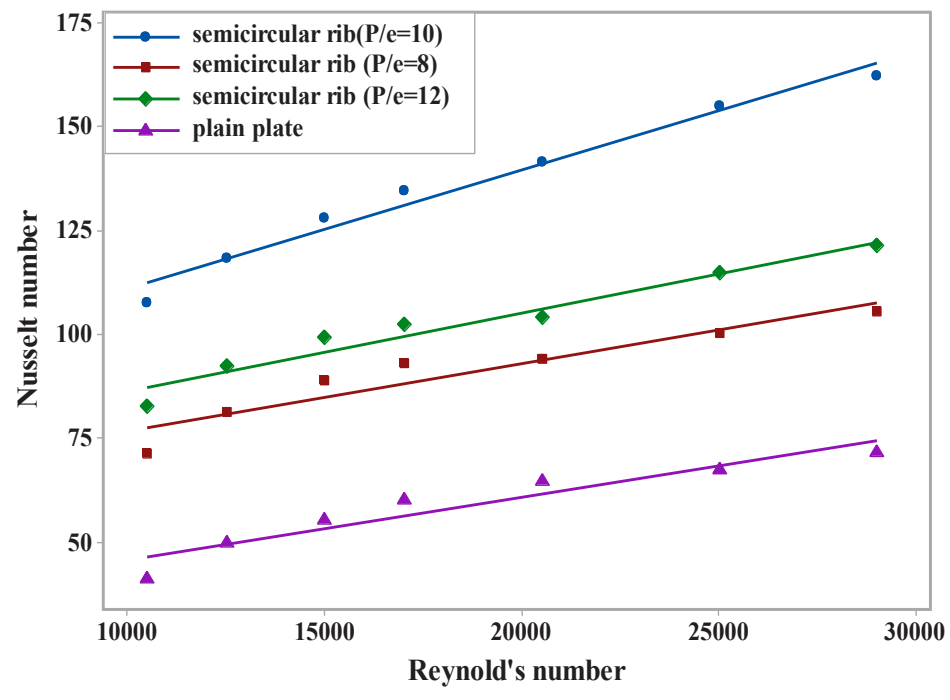

Figure 6. Reynold's number vs. Nusselt number for semicircular rib. 
In case of rib pitch $50 \mathrm{~mm}(\mathrm{P} / \mathrm{e}=10)$, the heat transfer was observed to be the highest, because the flow does reattach near the next rib, and the flow separation vortex and reattachment to rib wall play important roles in enhancement. Also, as seen in the distinct separation region and reattachment region between ribs, the flow strongly reattaches to the ribbed wall and detaches again quickly. In case of $50 \mathrm{~mm}$ spacing, the effective mixing between mainstream and fluids quite was observed near the walls, and secondary flow disturbs boundary layer growth on the side wall because the surface heat transfer is improved.

\section{Frictional Losses}

Ribs have enhanced heat transfer but also increased pressure drop, and generally, round edge ribs have less friction compared to sharp edge ribs. The friction factor for semicircular rib channel is presented in Fig. (7). Friction factor shows reducing trend as Reynolds number increased; this is because, the starting obstruction to flow is high, as the velocity increased retardation to flow reduced. The friction factor for the semicircular rib was greater than that of the plain plate because the large curvature shape of rib creates more turbulence, and also roughness component delays the flow and creates excess pressure drop. The semicircular rib has an average of $200 \%$ more friction compared to a plain plate, and semicircular rib with rib pitch ratio $(\mathrm{P} / \mathrm{e}) 8$ has more friction loss compared to other tested configurations. At rib pitch $40 \mathrm{~mm}(\mathrm{P} / \mathrm{e}=8)$, the distance between two ribs is less, and also the number of ribs on test plate increased because more retardation and disturbance were created in test plate; as a result of this, friction increased. We also observed larger separation zones and the additional form drag of having thicker boundary layers between the two ribs. When rib pitch is $60 \mathrm{~mm}(\mathrm{P} / \mathrm{e}=12)$, the number of ribs on test plate reduced, and a distance between two ribs is more because it offers smaller separation zones, and as a result, we found less friction compared to other tested cases. In case of rib pitch $50 \mathrm{~mm}(\mathrm{P} / \mathrm{e}=10)$, the channel offers medium friction means less than $40 \mathrm{~mm}$ rib spacing and more than $60 \mathrm{~mm}$ rib spacing; here, medium flow turbulence was observed because optimum distance was maintained between two succeeding ribs. For friction factor rib height was also an important parameter as rib spacing.

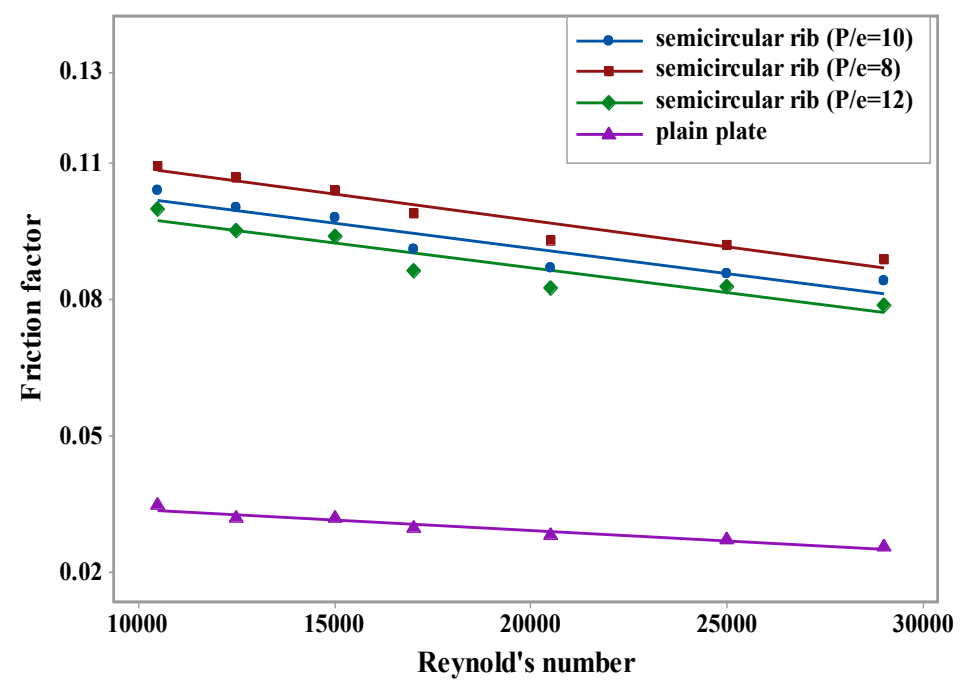

Figure 7. Reynold's number vs Friction factor for semicircular rib. 


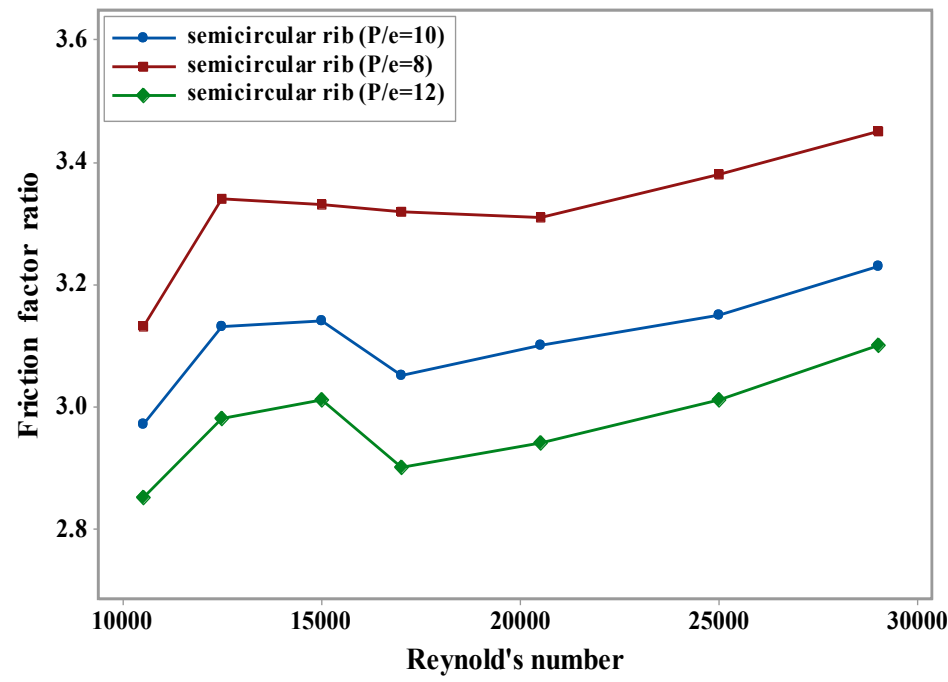

Figure 8. Variation of friction factor ratio against Reynold's number.

Fig. (8) shows the result of friction factor ratio for semicircular ribs. Friction factor ratio from 10000 to 15000 Reynolds number shows increasing trend after that decrease, but at Reynolds number 25000 onwards, it shows an increasing trend; this is because of the elimination of viscous sublayer with a rise in Reynolds number, and as the Reynolds no. increased, the boundary layer thickness decreased, causing an increase in the fluid velocities in the boundary layer. Semicircular rib with pitch ratio $(\mathrm{P} / \mathrm{e}=8)$ has more friction compared to other spacing ribs, enhanced 7 to $12 \%$ more friction compared to pitch ratio $\mathrm{P} / \mathrm{e}=10$ and 12 . Semicircular rib with $60 \mathrm{~mm}(\mathrm{P} / \mathrm{e}=12)$ spacing found less friction over 40 and $50 \mathrm{~mm}$ rib spacing because spacing between rib more retardation to flow becomes weaker because the friction was found to be less.

\section{Generalized Correlations of Experimental Data}

The least square method was used to generalize the current results, and also the Nusselt number ratio and friction factor ratios were correlates in terms of rib spacing ratio $(\mathrm{P} / \mathrm{e})$ and Reynolds number for the semicircular rib with different rib spacing configurations. The thermal efficiency index criterion was used to estimate the heat transfer enhancement of ribbed surfaces. The correlations for semicircular rib presented by Alfarawi et al. (2017) were used to generalize the experimental results. All the correlations are summarized below, and they are valid for a range of Reynolds number from 10000 to 29000, within a range of (P/e) from 8 to 12 for semicircular rib duct. For Nusselt number, a ratio correlation (9) was used, finding max. $\pm 13 \%$ deviation between experimental results and correlation results.

$\mathrm{Nu} / \mathrm{Nus}=10: 07 \mathrm{Re}^{-0.164}[\mathrm{P} / \mathrm{e}]^{-0.0595}$

The same way as that for friction factor ratio, correlation (10) was used, finding max. $\pm 10 \%$ deviation, and also thermal performance or efficiency index equation (11) was used, finding max. $\pm 9.65 \%$ deviation.

$$
\begin{aligned}
& \mathrm{f} / \mathrm{fo}=9.33 \mathrm{Re}^{-0.041}[\mathrm{P} / \mathrm{e}]^{-0.28} \\
& \eta_{\mathrm{i}}=4.73 \mathrm{Re}^{-0.15}[\mathrm{P} / \mathrm{e}]^{0.034}
\end{aligned}
$$




\section{Validation of Ribbed Duct Results Through CFD}

Experimental results of semicircular rib are validated through numerical results, and for simulation, the STARCCM version 2019 software was used. In this study, the realizable $\mathrm{k}-\varepsilon$ turbulence model was selected, and the results of turbulence model are validated by comparing its results with previous paper results. Figure 9 presents the top and side wall velocity contours of the semicircular rib, finding high turbulent kinetic energy appearing at the middle region between two adjacent ribs and in front of the ribs.

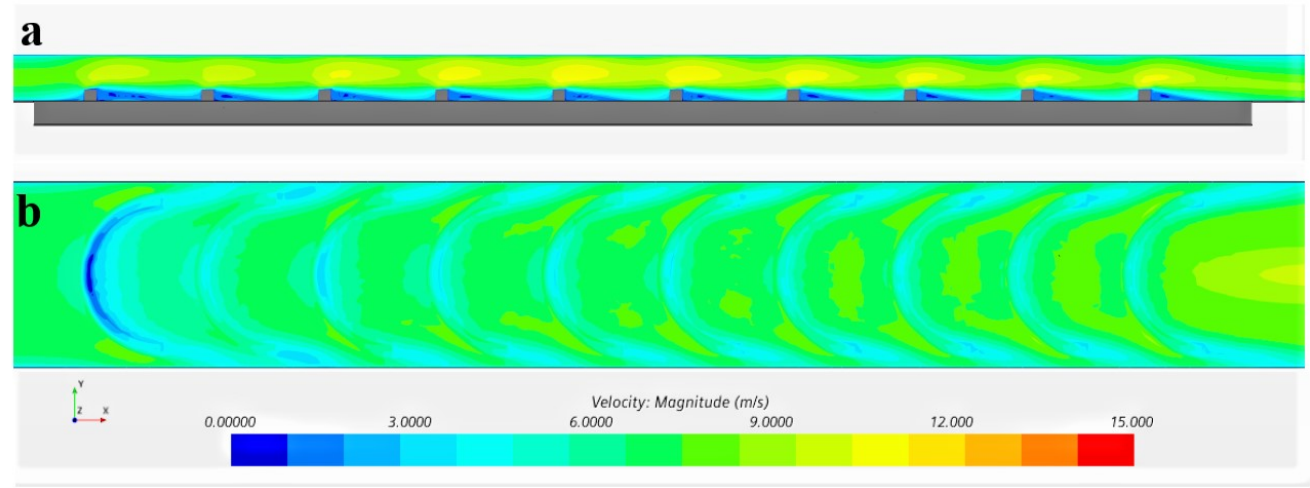

Figure 9. Side and top velocity contours of semicircular rib.

In the experimental results, semicircular rib with pitch ratio $(\mathrm{P} / \mathrm{e}=10)$ performed best, and the same results are observed in the simulation. Fig. 10 and 11 show variation in experimental and numerical results of semicircular rib $(\mathrm{P} / \mathrm{e}=10)$. From the fig., we found less variation between the numerical and the experimental results, and the differences for the Nusselt number and friction factor values between the numerical and the experimental results are less than $\pm 12 \%$.

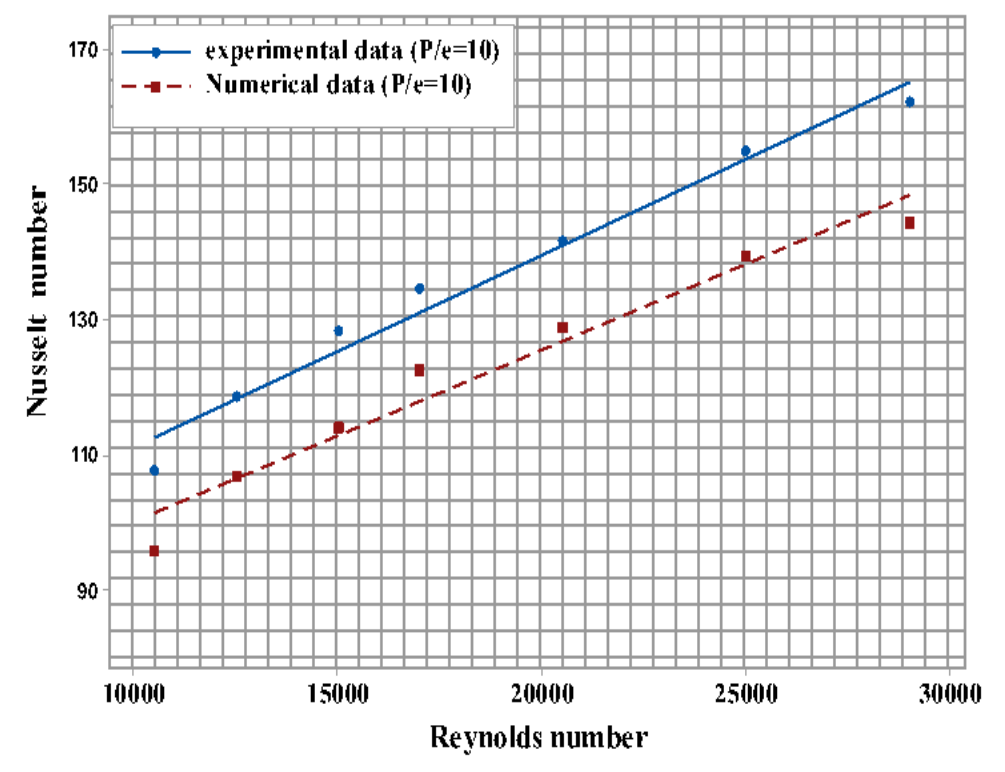

Figure 10. Variation of expt. and numerical results of Nusselt number. 


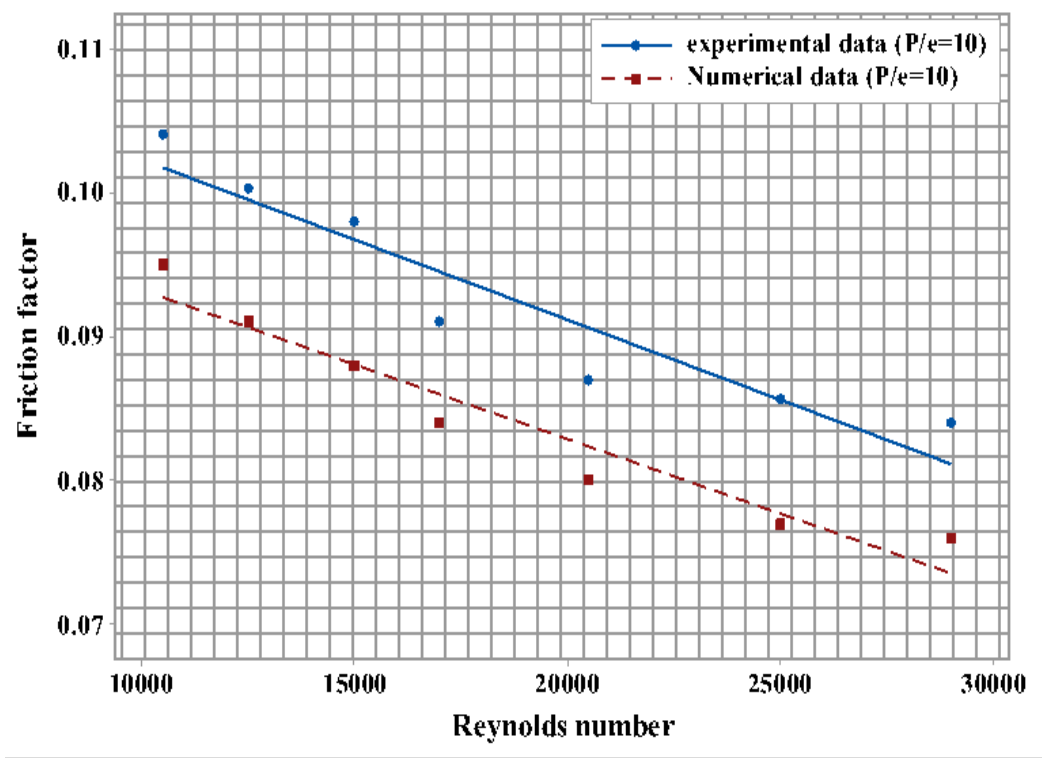

Figure 11. Variation of expt. and numerical results of friction factor.

\section{Thermal Performance (T.P.)}

For calculation of thermal performance, frictional losses are united with Nusselt numbers. Fig. (12) shows the thermal performance for all tested configurations. The semicircular rib with pitch spacing ratio (P/e) 10 offered the greatest thermal performance compared to all tested configurations. The semicircular rib with $\mathrm{P} / \mathrm{e}=10$ shows increased heat transfer enhancement and decreased frictional losses over the other rib spacing cases. Semicircular rib with pitch ratio $(\mathrm{P} / \mathrm{e})$ of 8 offered the lowest thermal performance compared to other rib spacing configurations.

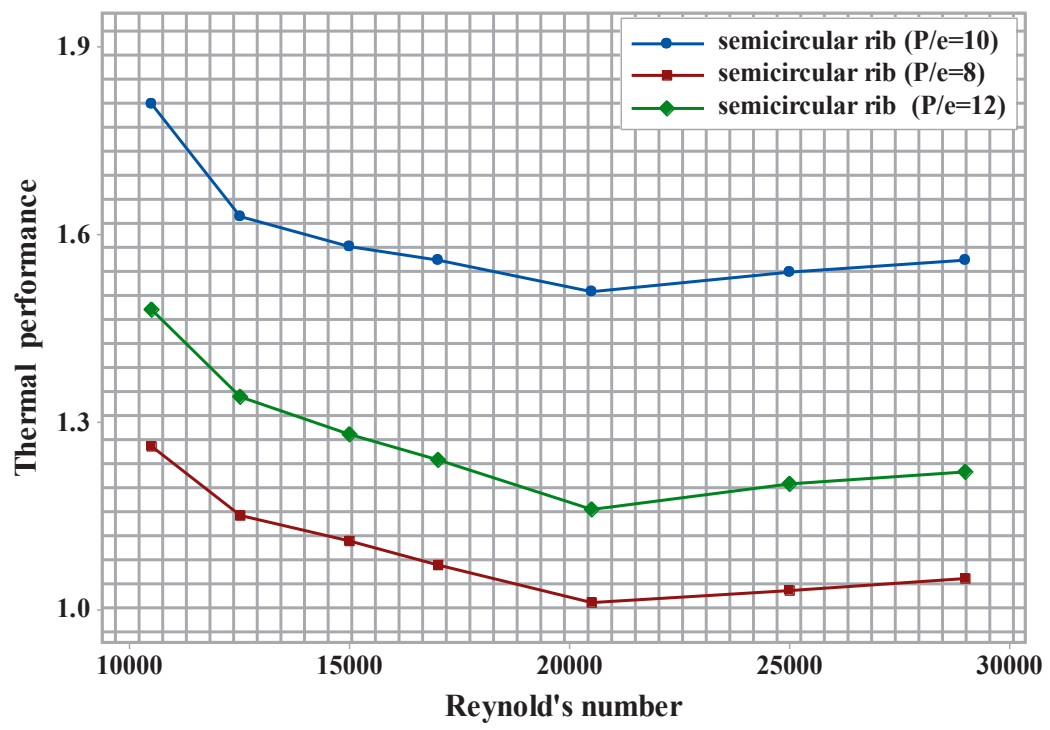

Figure 12. Reynold's number vs thermal performance. 


\section{CONCLUSION}

Heat transfer and flow friction characteristics in a rectangular duct with three rib spacing $(\mathrm{P} / \mathrm{e}=8,10,12)$ of semicircular rib were experimentally investigated. The experimental results propose the following conclusions:

- Semicircular rib performed better compared to plain duct, enhanced up to $130 \%$ heat transfer compared to plain duct.

- Semicircular rib with pitch to height ratio (P/e) 10 has highest thermal performance, found to be on average $40 \%$ heat transfer enhancement compared to rib spacing ratio of 8 and 12 .

- Friction in semicircular rib was on average $200 \%$ more than that of the plain plate, found to be the highest in semicircular rib with pitch to height ratio $(\mathrm{P} / \mathrm{e})$ of 8 compared to rib spacing ratio of 10 and 12.

- Semicircular shape rib with pitch to height ratio (P/e) 8 has the least thermal performance compared to other tested rib spacings.

\section{REFERENCES}

Luca Baggetta, Francesca Satta and Giovanni Tanda.2018. A Possible Strategy for the Performance Enhancement of Turbine Blade Internal Cooling with Inclined Ribs. Taylor \& Francis, heat transfer Engineering, DOI: 10.1080/01457632.2017.1421305.

Natthaporn Kaewchoothong, Kittinan Maliwan,Kenichiro Takeishi and Chayut Nuntadusit. 2017. Effect of inclined ribs on heat transfer coefficient in stationary square channel. Theoretical and Applied Mechanics Letters, 7: 344-350.

Jian Liu,Safeer Hussain,Jinsheng Wang,Lei Wang,Gongnan Xie and Bengt Sunden. 2018. Heat Transfer enhancement and Turbulent flow in a high aspect ratio channel (4:1) with ribs of various truncation types and arrangements. International Journal of Thermal Sciences, 123: 99-116.

Mi-Ae Moon,Min-Jung Park \& Kwang-Yong Kim.2014. Evaluation of heat transfer performances of various rib shapes. International Journal of Heat and Mass Transfer, 71:275- 284.

Niaben Zheng, Peng Liu, Feng Shan, Zhichun Liu and Wei Liu. 2016. Effects of Rib arrangements on the flow pattern and Heat transfer in an internally Ribbed heat exchanger tube. International Journal of Thermal Sciences ,101:93-105.

Anuj K Shukla and Anupam Dewan. 2016. Computational study on effects of rib height and thickness on heat transfer Enhancement in a rib roughened square channel .Sadhana, Indian Academy of Sciences, 41(6):667678.

Gongnan Xie, Jian Liu, Phillip M. Ligrani and Bengt Sunden.2014. Flow structure and heat transfer in a square passage with offset mid-truncated ribs. International Journal of Heat and Mass Transfer: 71, 44-56.

Wandong Bai, Wei Chen, Li Yang and Minking K. Chyu.2019. Numerical investigation on heat transfer and pressure drop of pin-fin array under the influence of rib turbulators induced vortices. International Journal of Heat and Mass Transfer: 129, 735-745. 
Govanni Tanda. 2011. Effect of rib spacing on heat transfer and friction in a rectangular channel with $45^{\circ}$ angled rib Turbulators on one/two walls. International Journal of Heat and Mass Transfer, 54:1081- 1090.

Govanni Tanda and Roberto Abram. 2009. Forced convection Heat Transfer in channels with Rib Turbulators inclined at $45 \mathrm{deg}$. Journal of Turbomachinery, 131:021012/1-10.

Weihua Yang,Shulin Xue,Yihong He and Wei Li.2017. Experimental study on the heat transfer charact- -eristics of high blockage ribs channel. Experimental Thermal and Fluid Science, 83: 248-259.

Guoqiang Xu,Yang Li and Hongwu Deng. 2015. Effect of rib spacing on heat transfer and friction in a Rotating two pass Square channel with asymmetrical 90 deg. Rib Turbulators. Applied Thermal Engineering, 80:386395.

Alok Chaube, Shailesh Gupta and Prakash Varma. 2014. Heat Transfer and friction factor enhancement in a square Channel having integral inclined discrete ribs on two opposite walls. springer Journal of Mechanical Science and Technology, 28 (5): 1927-1937.

Mohammad Ansari and Majid Bazargan.2020. Experimental and Numerical Investigation on Irregular Spacing of the Ribs in the Entry Length of a Solar Air Heater Channel. Taylor \& Francis, Science and Technology for the Built Environment,DOI: 10.1080/23744731.2020.1793639.

Yanlin Li,Yu Rao, Deqiang Wang, Peng Zhang and Xiangyu Wub .2019. Heat Transfer and pressure loss of turbulent Flow in channels with miniature structured ribs on one wall. International Journal of Heat and Mass Transfer, 131:584-593.

Naveen Sharma, Andallib Tariq and Manish Sharma. 2017. Experimental investigation of heat Transfer enhancement in Rectangular duct with Pentagonal Ribs. Taylor \& Francis Heat Transfer Engineering, DOI: 10.1080/01457632.2017.1421135.

Naveen Sharma, Md Shaukat Ali, Andallib Tariq and Manish Mishra.2018. Detailed heat transfer and friction factor characteristics in a rectangular duct with alternate solid and converging-slit ribs. Taylor \& Francis, Experimental Heat Transfer, DOI: 10.1080/08916152.2018.1463306.

S.Alfarawi, S.A.Abdel-Moneim and A.Bodalal. 2017. Experimental investigations of heat transfer enhancement from rectangular Duct roughened by hybrid ribs. International Journal of Thermal Sciences, 118: 123-138.

Kline S. J., and McClintock .1953. Describing Uncertainties in Single- Sample Experiments. Mech. Eng. (Am. Soc. Mech. Eng.) , 75: 3-8. 